\title{
Lautenistinnen in Deutschland im 18. Jahrhundert
}

\author{
by Per Kjetil Farstad
}

This article contains a descriptive presentation of some of the most outstanding female lutenists in the 18th Century German Countries: Luise Adelgunde Victoria von Gottsched, Anna Maria Wilhelmina von Althan Lobkovits, Christiane Mariane von Ziegler, Anna Ilsabe Lehmann Brockes, and Friederike Sophie Wilhelmine of Bayreuth. The article is a small contribution to the ongoing international female research within the area of musicology.

Der Artikel beschäftigt sich mit Lautenmusikerinnen in Deutschland im 17. und 18. Jahrhundert. In früheren Untersuchungen der männlich dominierten deutschen Lautenmusik kamen auch die eine oder andere Lautenistin zur Erwähnung, ${ }^{1}$ doch dieser Artikel gibt eine kleine Übersicht über die Personen und ihre Entwicklungsmöglichkeiten in diesen Zeiten.

\section{Die allgemeine Rolle der Lautenisten.}

Die Rolle eines professionellen Lautenisten im Deutschland des 18. Jahrhundert war die eines reisenden Musikers oder eines Angestellten bei Hofe. Es gab eine große Anzahl von Hofhaltungen in Deutschland zu Beginn des 18. Jahrhundert die ihre Musiker fest angestellt oder für kürzere Perioden angemietet hatten, um in kleineren oder größeren Hoforchestern spielen zu lassen und/oder Unterricht zu geben. In Kirchen oder Operhäusern spielten Lautenisten meist den Generalbass auf Lauteninstrumenten wie Theorbe, Chitarone oder zeitweise auch Gallichon ${ }^{2}$. Bei intimeren Anlässen beim Hofe, wurde auch Mandora ${ }^{3}$ oder die 11- bis 13 chörige Barocklaute solistisch, im Duett, zur Kammermusik oder zur Gesangsbegleitung gespielt. Die Lautenisten waren natürlich vollständig von ihrem Arbeitgeber abhängig und mußten die Musik schreiben und spielen, die diese wünschten. Die

1 (Farstad 1998 / Farstad 2000 / Farstad 2001 / Farstad 2002 / Farstad 2005). Vielen Dank an den Lautenforscher Joachim Domning für die Übersetzung des Artikels ins Deutsche und für die kritische Durchsicht des Inhalts.

2 Das Instrument wurde sowohl zum Solo- als auch zum Generalbaßspiel benutzt. Die Tabulatur zeigt normalerweise sechs Linien für sechs bis acht Chöre. Detaillierter: Farstad 2000, S. 110-114

3 Die Deutsche Mandora war ein der Lauten ähnliches Instrument und eine gute Alternative für alle die nicht die 11 bis 13-chörigen Instrumente, wie die Theorbe spielen wollten. Die vielen Tabulaturen für die Mandora im Deutschland des 18. Jahrhunderts. Zeigt deren Popularität. Normalerweise hatte sie sechs Chöre. 
Höfe, von einer herrschenden Elite gelenkt, bestimmte die Entwicklung und die Art des sozioökonomischen Systems ermöglichte die Kontrolle über die Städte und die Region ihrer Herrschaft. Dieses System hatte aber Vorteile für die Kunst, speziell für die Musik, denn die Rituale und die Zeremonien bei Hofe förderten eine reiche musikalische Aktitvität und damit Arbeit für Komponisten und Ausführende (Koskoff 2007).

\section{Die französische Mode und die Lebensumstände der Lautenisten}

Die Lautenisten waren hauptsächlich bürgerliche Musiker mit einem Status wie andere Bedienstete bei Hofe. Hiervon ausgenommen waren Künstler wie der bekannte Lautenist Silvius Leopold Weiss (1686-1750) in Dresden, Adam Falckenhagen (1697-1754) in Bayreuth und Ernst Gottlieb Baron (1696-1756) in Berlin. Auf Grund ihrer Popularität und hervorragenden Fähigkeiten bei der Komposition und instrumentalen Ausführung spielten sie in einer höheren Klasse als der Durchschnitt. ${ }^{4}$ Die Normalität für alle anderen Lautenisten des niedrigeren Standes war, dass sie mehrere Broterwerbe parallel ausüben mussten, ${ }^{5}$ z.B. als Kammermusiker, verantwortlich für das Kopieren von Noten, als Kammerdiener, Kammerherr, Türöffner oder als Ordner für die Kleider ihrer Dienstherren (Flotzinger 1986, S. 200-239) ${ }^{6}$.

In der Hauptsache waren die Lautenspieler natürlich für die Unterhaltung ihrer Vorgesetzten mit Musik zuständig, sie hatten zum Schlaf der Königin oder des Königs zu spielen oder den Könglichen Unterricht zu erteilen.

Die Lautenmusik war wie die Musik im allgemeinen mit der französischen Mode verbunden, die tonangebend für das adelige Deutschland zu Beginn des 18. Jahrhundert war. Trotz des Interesses an der italienischen Musik und dem wachsenden Einfluß der italienischen Oper blieb der französische Einfluß erhalten. Die Laute war mit dem aristrokratischen Leben eng verbunden, mit der

4 Die Popularität und die Wichtigkeit von S.L.Weiß läßt sich an seinem Gehalt aus der Anstellung am sächsischen hofe ablesen. Von 1717 bis 1734 erhielt er ein Geahlt von 1000 Taler pro Jahr. Ab 1734 wurde es auf 1200 Taler. Erhöht. 1736 schlug er die Offerte für 2000 Taler Jahresgehalt an den Hof nach Wien zu gehen aus. 1744 wurde sein Gehalt auf 1400 Taler erhöht, was ihn zum bestbezahlten Musiker bei Hofe machte (Volkmann 1906/07 S. 286-287; Neemann 1939, S.167 und 169; Smith 1977, S. 12; Hoffmann-Erbrecht 1986, S. 83).

5 Musiker wurden aber auch gerne mit Hofämtern betraut um die Gehaltskosten aus dem öffentlichen Haushalt bezahlen zu können, denn normaler weise wurden Musiker aus der „Schatull saliert“, d.h. von den Adeligen privat bezahlt. Adam Falckenhagen z.B. zusätzlich war als Kammerregistator bestallt.

6 Von 1723 bis 1726/27 war Adam Falckenhagen im Dienste von Christian in Weissenfels als Kammermusiker und Lautenist bei Hofe, zusammen mit seiner Ehefrau der Sängerin Johanna Äemilia Kegel. 
Wertschätzung und dem exklusiven Lebensstil, weil sie in dieser Umgebung den guten Geschmack, die Intimität und die Exklusivität verkörperte (Farstad 2000, S. 57-61).

\section{Frauen, Kunstmusik und Unterricht.}

Nur wenige weibliche Musiker finden sich in den Lexika und Geschichtsbüchern des 16. und 17. Jahrhunderts. Frauen waren von vielen öffentlichen Tätigkeiten wie z.B vom Singen in kirchlichen Chören ausgeschlossen. Das Bild änderte sich langsam im 18. Jahrhundert, speziell auch durch die Oper. In Italien erschienen im 17. Jahrhunderts die ersten Frauen auf den Bühnen der Comedia dell' arte. Mit der Einrichtung der kommerziellen Oper in Venedig 1637 öffnete sich die Tür weiter und mit der Ausbreitung der italienischen Oper im restlichen Europa des 17. Jahrhunderts boten sich mehr und mehr Möglichkeiten für Sängerinnen öffentlich aufzutreten. Selbst in Walters «Musicalisches Lexicon» von 1732 finden sich nur fünf Sängerinen. Charles Burney erwähnt mehrere Sängerinen und Komponistinnen (Franscesca Caccini und Barbara Strozzi). In Gerbers Historischbiographischem Lexicon der Tonkünstler (1790-92) finden sich schon zehn bis 15 weibliche Komponistinen. Eine davon ist Sophie Elisabeth, Herzogin von Braunschweig-Lüneburg, die 1651 eine Sammlung von Hymnen komponierte. Als Gegenreaktion auf die Ausgrenzung der Frauen beim Chorgesang in der Kirche eröffnete Johann Hiller 1771 in Leipzig eine Singschule für Damen. Bald darauf 1792 gründete C.F.C. Fasch ${ }^{7}$ seine Singakademie in Berlin, wo eine Arbeit für Musik mit gemischtem Chor stattfand (Tick \& Ericson 2007).

\section{Der Salon.}

In Frankreich und im Anschluß daran auch in Deutschland fanden Frauen über den Salon Zugang zum Musikleben (städtische und öffentliche Zusammenkünfte im privaten Heim) da es Frauen, wie bereits erwähnt, weitgehend verwehrt war in öffentlichen Orchestern mitzuwirken. Die Dichterin Louise Labe schrieb über ihre Salonschwestern: "Sie nehmen Federn und Lauten in die Hand, sie schreiben und singen aus ihrer Passion heraus!" Die Salons waren populär; sie fungierten als ein Ort, an dem Frauen ihre Kompositionen zeigen und aufführen konnten. Hier wurden Frauen entdeckt, soweit es «schicklich» war, und für das öffentlichen Musikleben rekrutiert (Tick \& Ericson 2007).

\section{Rollenmuster der Zeit.}

Noch 1787 benennt der Pädagoge Johann Daniel Hensel Stricken, Nähen, Kochen, Backen und Waschen als die wichtigsten Tätigkeiten einer Frau. Er bekräftigt damit

$7 \quad$ Fasch ist auch Komponist von Kammermusikwerken für Laute. 
die allgemeine Haltung gegenüber Frauen wie sie im 18. Jahrhundert in Deutschland üblich war. $\mathrm{Zu}$ den unwichtigeren, aber trotzdem nützlichen Beschäftigungen gehörte die Musik, die er in Instrumentalspiel und Gesang einteilte.

$\mathrm{Zu}$ der obersten Stufe indessen, der Musiktheorie und der Komposition konnten nur Männer aufsteigen (Rieger 1988, S. 53). Hierin muß einer der Gründe gesehen werden, warum so wenige Frauen aktive Komponistinen waren (Schusky 1997). Frauen könnten nicht so komponieren wie Männer lautete das Stereotyp, sie wurden diskriminiert. Ihre Kompositionen seien anders, geprägt durch die "schwache" weibliche Natur. Man meinte, dass diese Weitverbreitet war die Haltung der Männer, dass Frauen empfindsam und irrationell wären und man meinte, dass das Auswirkungen auf das Tun der Frauen zur Folge hätte. Jean-Jacques Rousseau (1712-1778) zufolge stand einer Frau kein Platz im öffentlichen Leben zu. Die Bedeutung der Frau wurde darin gesehen, dem Mann ein möglichst behagliches Leben zu bereiten und die kommende Generation aufzuziehen. Ihr Platz war das Heim. Das Ideal war die kleinbürgerliche Frau, die von den häuslichen Tätigkeiten absorbiert wurde und keine weiteren Interessen hatte (Aasen 2003). Am klarsten drückte es der Pädagogen Johann Heinrich Campe im Jahre 1789 aus:

Unter hundert preiswürdigen weiblichen [Ton] künstlerinnen, Zeichnerinnen... Tänzerinnen usw. findet man kaum eine die zur selben Zeit alle Pflichten einer guten Hausfrau und umsorgenden Mutter erfüllt (Rieger 1988, s. 41).

Solche Vorurteile und Einwände machten es Frauen schwierig in der Öffentlichkeit Raum für ihre Musik zu finden. Das Zitat berührt die Diskrepanz die zwischen den hausfraulichen Pflichten und den Ansprüchen einer Künstlerin besteht und die weitgehend ignoriert wurde. Die Äußerung Campes kann aber auch darauf hindeuten, dass es Frauen gab, die sich der Haltung ihrer Zeit widersetzen. Tatsächlich gab es dafür Vorbilder. Als Beispiel seien genannt: Anna Amalia, Prinzessin von Preußen, die Schwester von Wilhelmine von Bayreuth, oder Anna Amalia, Herzogin von Sachsen-Weimar und Sachsen-Eisenach (Nichte von Friedrich II und Wilhelmine). Beide komponierten bedeutende Musikwerke. Maria Antonia Walpurgis, Kurfürstin von 'Sachsen und Wilhelmine Markgräfin von Bayreuth komponierten beiden unter anderem Opern. Die Erwähnten gehören indessen zu den Ausnahmen ihrer Zeit. Sie hatten einen gute Ausbildung, Zugang zu den besten Lehrkräften in Ausführung und Komposition und waren finanziell unabhängig. Sie verfügten über Dienstmädchen die ihnen die Anforderungen als Mutter abnahmen und sie besassen die besten Kontakte zur Konzertszene (Schusky 1997, S. 10). 
Aber noch andere Begrenzungen kamen für Frauen hinzu, z. B. in der Auswahl der Instrumente. Der Philologe, Priester und Komponist Carl Ludwig Junker (1748-1797) sah Frauen am liebsten am Klavier oder mit Zither, Laute oder Harfe beschäftigt. Die Art und Weise des Spiels und sogar die Kleidung wurden genauso diskutiert, wie, dass eben bestimmte Instrumente nur von Männern bedient werden sollten. Als Konsequenz finden sich auch nur wenige Bilder von Frauen die andere Instrumente spielen. Selbst Flöte sollten Frauen nicht spielen (Schleuning 1984, S. 212), denn nur wer eine starke Brust hätte könnte dieses Instrument erlernen und vernünftig spielen (Hensel 1787, S. 20). Weiter schickte es sich nicht für Frauen Violine, Cello, oder wo möglich Horn Kontrabass, Fagott oder Trompete zu spielen. ${ }^{8}$ Diese Instrumente waren Männern vorbehalten. Darüber hinaus sollten Frauen nur Stücke spielen die nützlich oder vergnüglich waren (Schusky 1997). Das Resultat stellte sich dann so dar, dass leichte Kompositionen, oder leichter spielbare Stücke für Amateure und Liebhaber, gleichsam Stücke für Frauen waren und das Wort Dilletant ${ }^{10}$ blieb so stets verbunden mit "Amateur" oder "Weiblichkeit". Es wurde sogar gefragt, ob die Laute für Damen nicht ein zu schwieriges Instrument sein sollte? Sieht man die vielen Bilder lautespielender Damen aus dem 16. und 17. Jahrhundert, erweckt dies eher den Eindruck es wäre das favorisierte Instrument für sie gewesen. Thomas Mace diskutiert in seinem Buch "Musick's Monument" die Frage, ob die Laute ein schwaches, weiches Instrument und dadurch besonders weiblich sei. Wenn das so ist, sagt er, so sollte es nicht etwa nachteilig für die Laute sein, sondern vielmehr ganz im Gegenteil positiv. Mace wendet sich gegen das Stereotyp, dass die Laute ein kompliziertes Instrument sei, schwer zu halten als auch zu spielen (Mace 1676, S. 46). Zu beachten ist weiterhin dass während die Laute an Größe und Anzahl der Saiten vom 17. zum 18. Jahrhundert zunahm (was sie nicht nur für die Damen schwieriger machte), zwei neue Lauteninstrumenten in Deutschland im 18. Jahrhundert eingeführt wurden: das Gallichon und die Mandora. Die vielen Manuskripte für diese Instrumente zeigen dass diese hauptsächlich für Amateure geschrieben wurden und es ist anzunehmen, dass viele weibliche Lautenspielerinen (aber nicht nur diese) lieber ein einfacheres Instrument spielen wollten als die vielchörige Laute.

8 Erst jetzt sieht man in den Laienkapellen auch Trompeterrinnen oder Saxophonistinen.

9 Liebhaber: Ein Mensch der seine Liebe und Faszination auf eine speziellen Stil, oder Sache oder meistens der Kunst zu wendet; kann mit dem englischen Wort "fan" in Verbindung gebracht werden von "fantastisch". 


\section{Musikunterweisung bei Hofe.}

Generell gesagt, war die humanistische, Latein orientierte Universitätsausbildung gesellschaftlich in der Hauptsache für Männer reserviert, während die mehr "unsystematischen" Lehrfächer den Frauen vorbehalten blieben ${ }^{11}$ (Tick \& Ericson 2007).

Louise Gottsched z. B., so berichtet ihr Mann, hörte seine Vorlesungen im Nebenzimmer hinter der Tür versteckt. (Gottsched 1763)

Musikunterricht war bei Hofe leicht zugänglich, und Unterricht auf einem Musikinstrument, darunter Laute oder Gitarre, war ein Teil der "anständigen Unterweisungen", die adelige Hofdamen erhalten konnten.

Kunstausübung war generell ein Teil des täglichen Zeitvertreibes. Ein gewöhnlicher Tag im Leben einer Frau, die an einem italienischen Hofe um 1600 lebte, sah nach Pierre Erondell so aus:

Frauen....Wann kommt der Lehrer? Charlotte (die älteste Tochter): Der Tanzlehrer kommt um 9 Uhr, der Gesangslehrer, derjenige der uns auch das Virginalspiel lehrt, um 10 Uhr, der uns auf der Laute und der Viola da Gamba unterrichtet um 4 Uhr Nachmittags, und der Französisch-Lehrer gewöhnlich Morgens um 7 oder 8 Uhr (Endorell 1989). ${ }^{12}$

Das war sicherlich in Deutschland im 18. Jahrhundert ganz ähnlich. Der Musikunterricht der Kinder bei Hofe war Teil des Lebensunterhaltes der Musiker. Josef Haydn hatte in Wien z. B. freie Kost und Logis durch den Cembalounterricht der Marianne von Martinez (Tick \& Ericson 2007). Geht man durch die Geschichte der Höfe oder die relevante Literatur der verschiedenen Städte, findet man Auflistungen von Frauen des Adels oder der Aristokratie, die alle Lautenspielerinnen von gutem Rufe waren. Die folgende Aufzählung umfasst wahrscheinlich nur eine kleine Zahl der tatsächlich Laute spielenden Damen.

\section{Leipzig}

1750 war Leipzig das finanzielle Zentrum Deutschlands, zusammen mit Breslau,

11 Es sei auf das Lied "Ihr Schönen, höret an, legt euch auf das Studieren" in Sperontes singender Muse an der Pleiße 1736 verwiesen.

12 "Lady ... At what houres do your Maisters come? Charlotte [the eldest daughter]. Our dauncing Maister

commeth about nine a clocke: our singing Maister, and he that teacheth us to play the virginalles, at tenne; he that teacheth us on the Lute and the Violl de Gambo, at foure a clocke in the after noone: and our French Maister commeth commonly betweene seaven and eight a clocke in the morning". 
Danzig, Königsberg, Frankfurt/Main und Hamburg. Leipzig war keine Residenz, hatte aber als einer der größten Handelsorte eine Universität mit einem sogenannten "Collegium Musicum" aus der die erste wichtige öffentliche Konzertorganisation des 18. Jahrhunderts in Deutschland, das Gewandhausorchester erwuchs.

Viele prominente Persönlichkeiten der Laute waren mit der Universität assoziiert, z. B. Ernst Gottlieb Baron, Adam Falckenhagen, Anton Gleitsmann (Baron 1727/1976, S. 74) und Meusel (1727:72) ${ }^{13}$.

In Leipzig finden wir auch drei herausragende Lautenistinnen: Christiane Mariane von Ziegler (1695-1760), Johanna Eleonora Kropfganss (1710-?) und Luise Adelgunde Gottsched (1713-1762).

\section{Christiane Marianne von Ziegler}

Geboren in Leipzig, wurde sie am 30. Juni 1695 getauft und starb in Frankfurt an der Oder am 1. Mai 1760. Sie war eine gut ausgebildete Dame, die neben der Poesie der Musik zugetan war. Sie spielte Klavier, Laute und Flöte und war mit der Familie Gottsched in Leipzig verbunden. Sie lebte bis $1741 \mathrm{im}$ Hause ihrer Eltern (Romanushaus) in Leipzig und war eine sehr angesehene Frau. Ihr Salon war ein Platz, an dem sich Intellektuelle und Künstler in einem angenehmen Ambiente zum Spielen, Singen, Lesen und Diskutieren treffen konnten. Sehr wahrscheinlich hat auch J. S. Bach hier verkehrt.

1731 wurde sie Mitglied der "Deutschen Gesellschaft" ${ }^{14}$. Ziegler könnte mehrere Lautenlehrer in Leipzig gehabt haben, so z. B. Ernst Gottlieb Baron, der von 1715 bis 1719 in Leipzig lebte, oder Adam Falckenhagen, der von 1719 bis 1720 wechselweise in Leipzig und dann für die nächsten sieben Jahr im nahen Merseburg unterrichtete. Ziegler hatte durch ihre Bekanntschaft mit der Familie Gottsched vermutlich auch Kontakt zu Silvius Leopold Weiss, Johann Kropfganss und Johann Ludwig Krebs. Sie schrieb die Libretti für neun Bachkantaten 1725 (später publiziert in "Versuch in gebundener Schreibart"), BWV 68, 74, 87, 103, 108, 128, 175, 176 und 183. Da Johann Christoph Gottsched den Text zu Bachs Kantate "Auf, süss entzückende Gewalt", Trauer-Ode (BWV 183) von 1727 schrieb, kann die Bekanntschaft mit der Familie Gottsched Marianne Ziegler den

13 Andere Lautenisten mit Verbindungen nach Leipzig: Johann Gottfried Meley, (Lebensdaten unbekannt); Johann Kropfganss Sr. (1668-?); Johann Kropfganss Jr. (1708-ca. 1770); Johann Ludwig Krebs (1713-1780); David Hoyer (?-1720); David Kellner (ca. 1670-1748); Gotlieb Siegmund Jacobi (Lebensdaten unbekannt); Johann Christian Weyrauch (1694-1771); Schuchardt (Lebensdaten unbekannt); Setzkorn (Lebensdaten unbekannt); Rudolf Straube (1717-ca.1785); Maximilian Nagel (1712-1748); Ernst Gottlieb Baron (1696-1760); Johann Caspar Gleditsch (?-1747); Adam Falckenhagen (1697-1754); Guiseppe Maria Orlandini (1675-1760).

14 Die Deutsche Gesellschaft war eine Vereinigung von Literaten unter der Leitung von Gottsched. 
Weg zu dem Kontakt mit Bach geöffnet haben (Rifkin und Küster 2007). ${ }^{15}$

In ihrer Sammlung von Gedichten von 1728 "Versuch in gebundener Schreibart" pries sie die Musik und sprach von derer wichtigsten Funktion und über den Platz, den diese in ihrem täglichen Leben ausmachte: "Wie? Konnt ich mich denn nicht begnügen an meinem Flöt- und Lautenspiel, Das täglich mir zur Hand muss liegen?" Es ist wahrscheinlich, dass sie mit Luise Adelgunde Gottsched zusammen Laute spielte und an vielen Konzerten teilnahm, die im Hause Gottsched und ihrem eigenen veranstaltet wurden. In einer Widmung schrieb die Gottsched: “...auf Deinem Saitenspiel ein Muster reiner Lieder” (Gottsched 1763, S. 105). 1741 heiratete sie und zog nach Frankfurt /Oder.

Sie wird als eine umgängliche, freiweg sprechende, aber auch feierliche, soziale und intellektuelle Frau geschildert. Ein Zeitgenosse, Christian Gabriel Fischer (ca. 1730), schilderte, dass sie stets der Mittelpunkt bei sozialen und gesellschaftlichen Zusammenkünften war. Ihr Benehmen war so selbstverständlich, dass sie als "die Perle der Nation" bezeichnet wurde (Predeck 1727, S. 121-123). Durch ihre finanzielle Lage und ihre praktische Veranlagung konnte sie sich als Musikerin und Poetin profilieren. Sie entstammte einer wohlhabenden Familie, die Politiker, Advokaten und Richter aufzuweisen hatte. Ihr Vater, Franz Conrad Romanus (1671-1746) war Bürgermeister in Leipzig und ließ Marianne eine gute Ausbildung in Sprachen, Literatur und Musik angedeihen. Leider konnte sich bisher keine Lautentabulatur von ihrer Hand oder aus ihrem Besitz nachweisen lassen.

\section{Louise Adelgunde Victoria (Kulmus) von Gottsched (1713-1762),}

Louise Adelgunde Victoria von Gottsched (1713-1762) aus Danzig (Gdansk) hatte eine führende Rolle unter den Damen ihrer Zeit, nicht nur den Laute spielenden, und nicht nur Leipzigs, in der ersten Hälfte des 18. Jahrhunderts, inne. Die meisten der Frauen in Danzig wie auch in Leipzig waren, wie stets, Dilletanten oder Amateure, die Laute oder Mandora spielten. Sie begleiteten andere oder spielten unterschiedliche Tanzstücke. Das Lautenspiel waren sehr populär, sowohl unter den Adeligen wie auch unter den Bürgerlichen, und auch das Heim von Louise Adelgunde war ein beliebter Treffpunkt für einheimische wie durchreisende Künstler, darunter Lautenisten und Dichter. Oft wurden Konzerte in ihrem Heim veranstaltet. Luise selbst kam aus einem musikalischen Haus in Danzig. Ihr Vater spielte in seiner Jugendzeit Laute, ihre Mutter Klavier und Zister, und zeitweise wurden im Hause Kulmus Konzerte für Liebhaber veranstaltet.

Ein Sekretär namens Klein spielte Violine und ein anderer, namens Dr. Kade, spielte Laute bei vielen dieser Anlässe. Louise begann schon als Kind Laute zu spielen, so schrieb Gottsched (1763): "sie stahl sich oft weg, um auf der

15 Siehe auch Bülow (2007) und Schering (1941, S. 321-323). 
Bodenkammer, auf der alten Laute ihres Vaters, so wenig Saiten sie auch noch hatte, zu klimpern! "16 Weiter heißt es dort, dass sie Klavierunterricht erhielt, aber für die Laute kein Lehrer zu finden war. So hatte sie sich mit der Zeit das Spiel mehr oder minder selbst beigebracht. Jemand zeigte ihr die Noten (wahrscheinlich meint Gottsched hier Tabulatur, denn Noten kannte sie ja wohl vom Klavier) und Sayten und Griffe, konnte aber selbst nicht spielen. Dr. Kade hörte sie und erlaubte ihr, sich kleine Stücke vom ihm abzuschreiben. Und weiter:

"...Gleichwohl ersetze ihr Naturell alles übrige; so dass sie nachmals hier in Leipzig, die schwersten weißischen Stücke fertig, ja fast vom Blatt wegspielte; auch selbst dieses großen Meisters Beyfall erhielt, als er sie 1740 besuchte und ihr teils vorspielte, teils sie spielen hörte..." (Gottsched 1763).

Sie war Freundin und Schülerin von Weiss zugleich. Er sandte ihr eine Partita für Laute zusammen mit einem Brief, datiert vom 28. September 1741. Dieser Brief lässt auf eine engere Verbindung von Weiss mit der Familie Gottsched schließen, als man allgemein hin annehmen konnte. Dafür, dass Louise Gottsched an der Musik von Weiss interessiert war, gibt es glaubhafte Beweise, da einige Fantasien und Preludien von Weiss existieren, von denen wir annehmen, dass sie von ihrer Handschrift sind. Außerdem stammt zumindestens ein Teil der von Breitkopfs Verlagsdruckerei 1836 versteigerten Lautentabulaturen offensichtlich aus ihrem Besitz, denn sie tragen den Besitzvermerk: LAVG (Smith 1977, S. 22-23).

Aus ihrer "Lebensgeschichte, (Gottsched 1763) können wir vermuten, dass sie eine intellektuelle Frau war, die sich mit Musik und Poesie auskannte, selbst Bücher schrieb und ihre Zeit nutzte, um ihre eigene Musik sowohl für Klavier wie auch für Laute niederzuschreiben. Gottscheds Aussage: "sie schrieb ihre Noten selbst" kann man so deuten, dass sie selbst viele Stücke komponierte und/oder für die Laute selbst intavolierte. Sie spielte gut den Generalbass auf der Laute. Louise lernte ihren Mann 1729 in Danzig kennen; 1735 heirateten sie. Als sie dann nach Leipzig kam, studierte sie Komposition bei dem Bachschüler Johann Ludwig Krebs, welcher mit der Lautenszene Leipzigs wohlbekannt war (Gottsched 1763 Vorwort). Möglich, dass sie auch Lautenunterricht bei Krebs hatte. Zwei Briefe von Adam Falckenhagen zeigen, dass er in ihrem Hause Musik hörte und auch selbst gespielt hat ${ }^{17}$. Dass Louise Gottsched wirklich eine der intellektuellsten Frauen des 18. Jahrhunderts war, bekräftigte eine andere große Frau derselben Zeit, Maria Theresia, während des Besuches der Gottscheds in Wien.

Als ich [Louise] ihr [Maria Theresa] die Hand küsste, machte sie mir ein Kompliment. Sie sagte, sie hätte schon so viel Gutes von mir gehört. Ich war selbstverständlich

$16 \quad$ Gottsched 1763

17 Siehe die Briefe Falckenhagens an sie, Domning Falckenhagen Gesamtausgabe Bd. IV 
sehr berührt und gewaltig dankbar (Schonfeld 1908, S. 278-282).

Am nächsten Morgen hatte sie wieder eine Audienz bei Maria Theresa, die ohne weiteres aussprach, dass sie die gebildeteste Frau von Deutschland wäre (Schonfeld 1908, S. 278-282). Ihr Ehemann bringt in dem zitierten Buch auch einen Brief Louises an eine Freundin mit einem ausgedehnten Bericht über die Reise von Leipzig, Bayreuth (Besuch bei Wilhelmine und Falckenhagen?), Regensburg nach Wien und über die Audienz bei der Kaiserin. ${ }^{18}$

Sie war Dichterin, Essayistin, Übersetzerin und Theaterautorin. Ihr wichtigster Beitrag für die deutsche Literatur waren ihre Übersetzungen französischer Theaterstücke und ihre originalen Stücke im französischen Stil. Diese Stücke (5 insgesamt) ließen sie zur Mutterfigur des modernen deutsche Theaters werden. Vieles deutet darauf hin, dass Louise trotz der guten Anlagen ihr eigenes Talent in Literatur und Musik vernachlässigte und stets die "Dienerin" ihres Mannes Johann blieb. Als dieser mit der Arbeit an seinem deutschen Wörterbuch begann, beendete sie ihrer eigene literarische Tätigkeit. Johann Christoph empfing alle Ehre, Louise erledigte den größten Teil der Arbeit, welche über 30 Jahre von morgens bis abends andauerte, und so war sie frühzeitig verschlissen. Als sie dann krank war, bemerkte ihr Mann öffentlich, dass sie nicht mehr höflich reagierte, wenn er sie fragte, wann sie denn an seinen Manuskripten weiterarbeiten würde. Sie starb mit 59 Jahren. Es ist sehr wahrscheinlich, dass sich alle ihre ausgezeichneten Anlagen als Musikerin in diesem Umfeld nicht entwickeln konnten, und vielleicht haben wir hiermit eine der größten Lautenistinnen der Geschichte verpasst. In dem nach ihrem Tode von Gottsched veröffentlichten Buch mit Werken seiner verstorbenen Frau sind auch Kondolenzbriefe aus aller Welt enthalten, und auch hier tauchen immer wieder Bemerkungen über ihre musikalischen Fähkeiten auf.

\section{Johanna Eleanora Kropfganss}

Johanna Eleanora Kropfganss (geboren am 5. November 1710 in Breslau) war die Tochter von Johann (Gottlieb) Kropfganss Sr. und die Schwester von Johann Kropfganss (geb. 1708) sowie Johann Gottfried Kropfganss (geboren am 17. Dezember 1714). Alle drei Kinder spielten Laute. Johann transponierte und spielte den Generalbass bereits mit acht Jahren prima vista, er komponierte auch sehr gut. Johanna Eleanora war eine sehr gute Lautenistin, und es ist nicht zu bezweifeln, dass sie von ihrem Vater auf der Laute unterrichtet wurde. Nach Walther war sie eine Lautenistin, die sowohl den Hohen wie den Verständigen wohl gefiel (Walter 1732, S. 346-347). Es ist gewiss, dass Johanna Eleanora bei Versammlungen Konzerte spielte. Trotzdem finden sich keine Kompositionen wie auch keine nachweisbaren Lautentabulaturen von ihrer Hand.

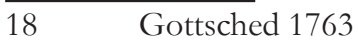




\section{$\underline{\text { Wien }}$}

Anna Maria Wilhelmina [e] von Althan Lobkovitz. (1703-1754)

Sie war eine tüchtige Lautenistin und Schülerin von Sylvius Leopold Weiss. In einem Brief an Fr. Alagrotti schrieb der Komponist und Geiger Guiseppe Tartini (1692-1770), dass er nicht zwischen dem Lautenspiel von Weiss und der Prinzessin Wilhelmina unterscheiden könne (Klima 1984, S. 23). Weiss widmete ihr einige Werke $^{19}$.

Anna Maria war die zweite Frau von Philipp Hyazinth Lobkovitz (16801734). Philipp war ein begeisterter Lautenist und Komponist sowie ein persönlicher Freund von Silvius Leopold Weiss (Volkmann 1906/1907, S. 281-282). Von seinen Reisen nach England, Frankreich und Italien brachte er Ladungen voll Musik mit nach Hause, darunter viele Lautenkompositionen. Er bestellte sich Kompositionen von Weiss, und in einem Brief an Phillip, datiert vom 17. November 1728 verspricht Weiss ihm bei einer anderen Gelegenheit einige Lautenstücke zu senden (Volkmann 1906/07, S. 281-282). Jiri Tichota schrieb eine mit CW signierte Sarabande der Comtese Wilhelmine von Althan zu. Das Werk befindet sich in der Ms. II Kk 78. der Familienbibliothek der Familie von Lobkowitz, welche einen wichtigen Teil der Gitarren- und Lautenmanuskripte des 17. und 18. Jahrhunderts enthält. Phillip war wahrscheinlich auch der Sammler der Lautenkompositionen von Laurent (JacquesAlexandre) de Saint-Luc, (1663 bis nach 1700). Lobkowitz' Name ist übrigens auch mit der Handschrift Ms. Suppl. Mus. 1078 in Wien Nationalbibliothek (Meyer 1997 III/1, S. 138) verbunden. ${ }^{20}$

Nach der Flucht in seinen Palast in Wien 1729 veranstaltete die Familie Lobkowitz-Althan dort regelmäßig Konzerte, und prominente Musiker und Sänger hatten bei ihren Besuchen in dem Haus häufig Auftritte. Als Fürst Phillip Lobkowitz $^{21} 1734$ starb, war Wilhelmina 31 Jahre alt. Man kann davon ausgehen, dass sie auch weiterhin Laute spielte und Musikalien sammelte. Christian Heege, der Bibliothekar am Karolinischen Kollegium war, verfasste 1727 eine Lobpreisung für die Fürstin Wilhelmina und beschrieb darin deren große Künste auf der Laute:

Was, sag ich, eine Nachtigall. Durch Schlagen, Aechzen, Ziehen, durch Steigen Fall und Schall. Vermag beweglich auszuüben, Die steckt in

19 Siehe auch (Reilly, Smith und Crawford 2007).

20 Phillip war der älteste Sohn von Prince Ferdinand August Leopold Lobkowitz. Die adelige Familie von Lobkowitz war sehr interessiert an Kunst und speziell an Musik. Philip stand freundschaftlich zu Weiss und war in der Wiener Lautenszene gut bekannt.

21 Weitere Anmerkungen zur Familie Lobkowitz und Lautenmusiken in Verbindung zur Familie siehe Farstad (2000). 
Wilhelmines Trieben. Sprich Schönheit! (Auch hier muss selbst Wilhelmine sprechen!) Sprich Tugend! (Wilhelmine muss hier das Schweigen brechen!) Sprich Klugheit, sprich von unserer Herzogin. Du schweigst. Sie bleibet selbst Ihr Zeugnüs und Gewinn. Und endlich sprich, geschwätzig Lautenspiel! Bald legt sie auf der Laute Leitern an, Und steig in einem Blitz vom Himmel zu der Erde, Bald singt der Griff, bald rauscht der Klang. Mit zitterndem, vermischeten Gesang, Mit englischer volkommener Gebehrde, Aus todten Sayten bringt sie in gedehntes Fühlen. Ihr Finger spricht: Halten! im Spühlen. Alle Finger Werden Springer! Und die Laute ist eine welt Wo das Regen Und Bewegen Wilhelmin in Händen hält. Jetzt circult sich ein Wirbelwind, Jetzt kömmt ein Sturm, nun machts die Fürstin helle, Jetzt zäumet sie das Meer, Jetzt wirft sie eine Welle, Jetzt geht es lieblich hart, jetzt schmerzlich und gelind. Die Laute stirbt in Weisischen Tombeau ${ }^{22}$, Die Fürstin rührt sie an, so lebt sie und wird froh. O Hand voll Kunst, o Hand ohn ihresgleichen! Wer kunt', wer kan, wer wird an Wilhelminen reichen? Jetzt nimmet Sie das gründliche Clavier, Schickt tausend Läuffer aus, kömmt eher als die tausend, Nun schmeichelt das Piano, nun ist das Forte prausend; Sie spant mit der volkommen Hand Mich die Music, in schönste Fesseln ein... (Klima 1984, S. 36-38).

Das war natürlich eine übertriebene (panegyrische) Lobpreisung ihrer künstlerischen Fähigkeiten als Lautenistin, wie sie in der damaligen Zeit üblich war. Solch ein Gedicht kann selbstverständlich nicht als wahrheitsgemäße Aussage begriffen werden, doch zeigt sie, im Einklang mit anderen Aussagen, eine technisch wie musikalisch auf professionaler Höhe stehende Künstlerin. So stellt sich die Frage, ob ein normaler Lautenist an sie heranreichen konnte. Das stimmt ja auch mit Tartinis Aussage mit überein, die besagt, dass sie mit Leopold Sylvius Weiss, Deutschlands größtem Lautenisten des 18. Jahrhunderts, auf gleicher Höhe stand. Tartini hatte es nicht nötig, aus politischen Gründen zu schmeicheln und kann als wahrhaftiger Zeuge dienen und damit die Aussage von Heegs stützen. Dem Gedicht zu Folge, konnte sie beim Publikum die Affekte und Passionen wecken, die das oratorische Ideal forderte, dass durch die griechische Rhetorik, die im 18. Jahrhundert den Weg in die Musik fand, inspiriert war. Das brachte den "galanten Ausdruck" mit "einiger Eleganz" und dem "Gebrauch von Affekten" mit sich (Baron 1727/1976, S. 117).

Die oben erwähnte Aussage kann bedeuten, dass Wilhelmina diese Kunst beherrschte. Das heißt im übrigen nicht, dass sie komponierte, und es sind auch keine Musikstücke von ihr überliefert, sieht man von der Zuweisung Tichotas ab

22 Anspielung wahrscheinlich Weiss,'Tombeau Sur La Mort De M. Comte De Logy"? 
(Klima 1984, S. 23).

\section{Praha}

Frau von Mestel

Wir wissen nicht viel über Laute spielende Frauen in Prag, nur Paul Nettl erwähnt eine Frau von Mestel, die eine geschickte Lautenistin gewesen sein soll. (Nettl 1922-23, S. 162). ${ }^{23}$

\section{Königsberg}

Zwei Musiker, die Frauen in Königsberg unterrichteten, waren Johann Reichardt und C. G. Tauscher. ${ }^{24}$ Reichert war ein respektierter Lautenist und Lautenlehrer, der Caroline Amalie Truchsess-Walburg (1727-1791), die später in Königsberg Gerhard Johann Keyserlingk heiratete, unterrichtete. 1755 kaufte die Gräfin ein Haus in Hestehagen in der Nähe des Palais Keyserlingk. In diesem Haus unterrichtete Reichardt auf seiner Laute. Sein junger Sohn Johann Friedrich Reichardt spielte Violine, und schon mit jungen Jahren musizierte er zusammen mit der Gräfin auf Violine und Laute verschiedene Male in den angesehensten Salons.

Frau Waga, Hoffmanin und Ganswindt, und die Ehefrauen von Bertramm und Hess. Kosack erwähnt, dass C. G. Tauscher (ca. 1740 in Königsberg gestorben) die meisten der herausragenden einheimischen wie auch fremde Lautenisten unterrichtete, darunter Bielogradski, der später nach Dresden reiste. Andere seiner Schüler waren Hofrat Reinhold Hoyer und die Frauen der Hofsekretäre Bertramm und Hess. Das Hoyersche Haus lag in Hestehagen in der Nähe des gräflich Keyserligkschen Palais. Weitere Frauen waren die Tochter des Tribunals- und Hofgerichtsrats D. Waga; so wie die Ehefrau von einem Hoffmann und Ganswindtin, Ehefrau von Ganswindt (Kosack 1935, S. 56 und Lauson, 1753 S. 143-145).

Alle diese Frauen gehörten genau wie die übrigen zur Elite der Stadt, und deren wichtigster Zeitvertreib war das Lautenspiel und die Musik. Lausons Gedicht zu Folge (siehe unten) kann man einen Eindruck von dem Interesse an dem Musikschaffen und den besonderen Lebensstil bekommen. Lauson erwähnt

23 Es gab viele männliche Lautenisten und Lautenkomponisten in Praha: Philipp Hyazinth Lobkovitz (1680-1734); Jakob Karl Kohaut [Kohott] (ca.1678-1762); Schlinsky (Lebendaten unbekannt); Johann Anton Losy von Losinthal (1650-1721); Johann Adam (Graf) von Questenberg (1678-1752); Aurius Dix (1669-1719); August Wilhelm Heinrich Gleitsmann (1698-?); Antonius Eckstein (ca. 1657-1722); Georg Adalbert Kalywoda (Lebensdaten unbekannt) (Zuth 1926-28, S. 152); Ivan Jelinek (16831759); Jan Jiri Neruda (1707-1780); Häussler (Lebensdaten unbekannt); Antoni Ceccherini (Lebensdaten unbekannt); (Wenzel?) Cqierwenka (Lebensdaten unbekannt); $P$. Amandus (Lebensdaten unbekannt) (Farstad 2000).

241745 bekam er Geigenunterricht von Franz Benda und Lautenunterweisung von dem russischen Lautenisten Timofei Bielogradsky (Kosack 1935, S. 56). 
Tauscher als einen der gegenwärtig berühmtesten Virtuosen auf der Laute, und dieser scheint ein inspirierender Lehrer gewesen zu sein (Lauson 1753, S. 143).

Mehrere Damen in Königsberg

Außer den Frauen, die schon erwähnt wurden, können wir in dem genannten Gedicht von Lauson "Die Laute", in dem Johann Adolf Faustinus Weiss (1741, Dresden bis 1814, Dresden) erwähnt wird, den Eindruck gewinnen, dass noch andere Frauen in Königsberg Laute spielten:

Jetzt spielt der Drollichte Weiss auf seiner lockeren Laute, Und schaft für Königsberg Dressdensche Lust.

Er reitzt den silberen Ton mit ausgestopfeten Fingeren, Uns jedes Grübchen am Finger spielt mit.

Er lehrt die Schönen der Stadt die Kunst recht spielend zu leben.

Frau Waga, Frau Hoffmann und Frau Ganswindt spielten.

Die Musen die ihm dankten, waren meist singenden Schönheiten.

Seit dem Tode seines Vaters Silvius Leopold Weiss lebte Johann (von ungefähr 1750-1757) im Hause des Grafen Keyserlingk in Königsberg. Hier unterrichtete er viele junge Frauen der besseren bürgerlichen Klasse der Stadt im Lautenspiel. Keyserlingk war S. L. Weissens Beschützer und bezahlte Johanns Musikausbildung.

\section{Mannheim}

\section{Juliana Margaretha Weiss}

Die Wittelsbacher Hofkapelle in Düsseldorf, Heidelberg und Mannheim war das Lautenzentrum der Weiss-Familie, die von einem Ort zum anderen im Dienst als Hofmusiker zog. ${ }^{25}$ Das waren der Vater (1686-1750) von Silvius Leopold Weiss (1686-1750) und sein anderer Sohn Johann Sigismund Weiss (um 1690-1737) und die Tochter Juliana Margaretha Weiss (geboren am 21.09.1690, gestorben am 20.11.1765). Während Silvius Leopold schon bald nach Dresden ging, blieb der Rest der Familie den Wittelsbachern treu. Über Juliana Margaretha wissen wir leider bisher noch recht wenig. Neemann schrieb, dass sie die einzige Tochter von Johann Jakob und Anna Margaretha war, und Baron erwähnt die Schwester mit folgenden Worten:

Die beyden herrn Söhne, herr Silvius Leopold und herr Siegmund Weiss, nebst ihrer Schwester, welche nunmehro mit einem Priester in der Pfalls verheirathet, haben dieses Instrument vollend auf den höchsten Grad der

25 Thomsen-Fürst, Rüdiger, Lautenisten und Lautenmusik am kurpfälzischen Hof in Mannheim, Jahrbuch der dt. Lautengesellschaft Nr.VII/2003 
Vollkommenheit gesetzt (Baron 1727/1976, S. 70).

Nach dieser Aussage können wir davon ausgehen, dass Juliana, ganz im Geiste der Familie Weiss, eine gute Lautenistin war und auch aktiv am Hof mitwirkte. Mit 30 Jahren heiratete sie Johann Lorenz Weiss, einen Pfarrer aus Großkarlbach in der Pfalz. Die Jahre bis zu ihrer Hochzeit verbrachte sie mit ihren Geschwistern vermutlich im Haushalt ihrer Eltern. Nach der Heirat zog sie 1722 nach Rheingönheim (heute ein Ortsteil von Ludwigshafen), und ab 1733 lebte sie in Weinheim an der Bergstrasse (Thomsen-Fürst S. 41-47).

\section{Dresden}

\section{Prinzessin Maria Antonia Walpurgis Wittelsbach (1724-1780}

Eine weitere mit der Laute verbundene Frau war Prinzessin Maria Antonia Walpurgis von Wittelsbach, die älteste Tochter von Karl Albert von Bayern (später Karl VII.) und Maria Amalia von Österreich. Sie wurde mit dem Kurprinzen Friedrich Christian 1747 in Dresden verheiratet. Sie war eine gute Sängerin, Cembalistin und Lautenspielerin. Ihren ersten Musikunterricht erhielt sie in München. Nach ihrer Hochzeit setzte sie ihre Studien in Dresden bei Nicola Porpora und J. A. Hasse fort und beschäftigte sich speziell mit der italienischen Oper. Sie trat als Sängerin und als Klavierspielerin auf, und der Musikhistoriker Charles Burney war begeistert von ihrem Gesang. Über längere Zeit nahm sie auch Unterricht auf der Laute bei S. L. Weiss (Fürstenau 1971, S. 188).

Am 5. Oktober 1747 schrieb ihre Mutter, Kaiserin Amalie, einen Brief an sie, in dem sie um Stücke oder eine Partita von Weiss für ihre dreizehnjährige Tochter (Maria Antonias Schwester) bat. Amalia verglich Setzkorn ${ }^{26}$ mit Weiss und versicherte, dass die Stücke von Weiss mit dem rechten Geschmack komponiert seien, im Gegensatz zu den "Kratzereien" Setzkorns (Neemann 1939, S. 169).

Es existiert ein Gemälde mit dem bayrischen Hof von Peter Jakob Horemans, das im Schloß Nymphenburg hängt. Die Malerei zeigt die kurfürstliche Familie und die Sachsen, die mit dem Musizieren und Kartenspiel beschäftigt sind ${ }^{27}$. Maria Antonia Walpurgis war bei diesem Ereignis anwesend. Auf der rechten Seite des Bildes ist eine Barocklaute mit Bassreiter zu sehen, vermutlich ein 13-chöriges Instrument. Die Laute kann ihr zugeordnet oder aber auch mit ihrer Schwester in Verbindung gebracht werden. Nach dem Siebenjährigen Krieg und dem Tod vom Kurprinz Friedrich 1763 ging das Kulturleben in Dresden zurück. ${ }^{28}$

\footnotetext{
$26 \quad$ Setzkorn war von 1744 bis 1750 in Leipzig.

27 The New Grove, 1980 (reprint 1995), vol. 7, S. 274

28 Zwischen 1763 und 1779 korrespondierte Maria Antonia mit Friedrich dem Großen in Berlin. Aus den Briefen geht hervor, dass sie sowohl persönlich als auch musikalisch in einer ziemlichen Isolation lebte. Als Komponistin schrieb sie damals zwei Opern: Il trionfo della fedeltà (Sommer 1754, Dresden) und Talestri,
} 
Werke für Laute von ihrer Hand hat man bisher nicht gefunden. Zwischen den persönlichen Sachen, die sie 1747 mit nach Dresden brachte, befanden sich u. a. auch "14 Stück Hassischer Opern Arien auf die Laute". ${ }^{29}$ Die Lautenstimmen sind kurz gesagt Generalbassaussetzungen. Die Handschriften stammen von einem Kopisten oder Schreiber aus München, aber man weiß nicht, um wen es sich handelte. Zwölf von diesen Arien stammen aus der Oper "Cleofide", welche im Jahre 1731 in Dresden aufgeführt wurde. Das originale Manuskript dieser Oper befindet sich in der Dresdner Landesbibliothek, darunter ein gesondertes Stimmheft für Theorbe. Als Theorbist kann bei der Ausführung entweder Gottfried Bentley oder Sylvius Leopold Weiss mitgewirkt haben. Es ist sehr wahrscheinlich, dass das Manuskript gleich nach der Aufführung von Dresden nach München kam. Es ist nicht unwahrscheinlich, dass Maria Antonia die Generalbass-Stimmen dieser Arien gespielt hat. Sie hatte lebhaftes Interesse an Opern und darüber hinaus muss sie eine fähige Lautenspielerin gewesen sein.

\section{Hamburg}

Anna Ilsabe Lehmann

Johann Mattheson klagt in seinem Buch "Das Neu-Eröffnete Orchestre" (1713) über die vielen dürftigen Lautenisten in seinem Umkreis, aber er preist Weiss und nennt eine fähige Lautenistin in Hamburg - "ein gewisses artiges hiesiges Frauenzimmer/ mit Verwunderung und zur Genüge beweisen kan" (Mattheson 1713, S. 276).

Der Lautenforscher Tim Crawford (Crawford 1986, S. 30-31) nimmt an, dass es sich bei dieser Dame um Anna Ilsabe Lehmann handelt, die berühmt war für ihr Lautenspiel. Ihr Mann, der Dichter Barthold Heinrich Brockes (16861747), war Ratsherr in Hamburg. Brockes heiratete Anna Ilsabe Lehmann 1714, die eine gute und vernünftige Frau war. Brockes sagt selbst, dass unter den guten Eigenschaften Ilsabes vor allem ihre Vollkommenheit in der Musik, darunter ihr Lauten- und Klavierspiel und ihr Gesang, hervorstachen. Im Alltag lebte sie zurückgezogen und widmete sich gern dem Haushalt und zog ihre Kinder auf. Brockes erwähnt besonders ihr Verhältnis zu Gott und ihre wahre Gottesfurcht (Lappenberg 1847, S. 207-208).

Barthold Heinrich Brockes' Bekanntenkreis bestand sowohl aus Literaturwie auch aus Musikfreunden, und Musik und Literatur war wohl auch das enge Band zwischen Mitgliedern dieses Kreises. In einem Gedicht, das Brockes für seine

regina delle amazzoni (6. Februar 1760, München, Nymphenburg), auch die Libretti schrieb sie selbst. Viele Kompositionen in dem Dresdener Manuskript tragen ihren Namen, aber man ist sich nicht sicher, ob diese Werke wirklich von ihr sind oder diese nur ihren Namen aus Repräsentationsgründen tragen.

Musikbibliothek der Stadt Leipzig, Ms. III. 46. a 
Freunde schrieb, pries er Musik und Lautenspiel seiner Anna Ilsabe. Das Gedicht trägt den Titel " Die Laute der Belisa besungen von Belisander".

Belisa war der Rufname Anna Ilsabes, ${ }^{30}$ für diejenigen, die Brockes näher kannten. Das Gedicht enthält auch eine Anerkennung der Musik von Georg Friedrich Händel. Es erscheint deutlich, dass Anna Ilsabe Musik von Händel auf ihrer Laute spielte, entweder intavolierte sie Händels Musik für andere Instrumente oder sie spielte originale Lautenmusik von ihm. Hier ist Brockes' Lobpreisungsgedicht über Anna Ilsabes musikalische Begabung und ihr vorzügliches Lautenspiel:

"Sobald ihr Ton durchs Ohr uns sanft die Herzen schlägt /

Kocht gleich ihr Blut und wallt, die Seele wird bewegt, Und steig verwunderungsvoll aus uns'rer engen Brust /

Auf Leitern der Music ins Paradies der Lust, Wo, gleichsam von der Welt Getümmel abgeschieden /

Sie voller stolzen Ruh, ergötzt, vergnügt, zufrieden /

Von jedem Ton erquickt, entzückt durch jeden Schlag /

Aus Furcht, sie misse was, kaum Atem holen mag.

Den Saiten muss an Klang ein Glöckchen weichen /

Dahero, weil ihr Ton so hell und rein, so zart"

"So scheints, ob Ihre Hand auf unsichtbare Ahrt /

Ein himmlisch Glocken-Spiel mit güld'nen Strickchen zöge,

Und ob die schlanke Hand nicht sprünge, sondern flöge.

Die Sinne folgen Ihr, die Herzen fliehn und stehn,

Nachdem die Fluchten hoch, schnell oder langsam gehn.

Bald singt ein Griff allein, bald rauscht der Töne Menge.

Und macht in Luft und Ohr ein lieblisches Gedränge

Von lauten Cirkelchen; bald teilt Sie Mass' und Zeit /

In unbeschreiblicher geschwinder Fertigkeit /

Mit Regel-mässigen unzäligen Manieren /

Oft drohet Sie mit Fleiss, den Wollaut zu verlieren, In einem falschen Ton; doch zeucht die kluge Hand /

Aus einem harten Klang und herben Gegen-stand /

Noch süss're Zärtlichkeit. Will Sie aus Hendels Stücken /

Mit einem sanften Satz des Hörers Ohr erquicken:

So greift ein jeder Griff ihm so die Sinnen an, /

Dass er den Wunder-Ton nicht g'nug bewundern kann.

Die Saiten weiss Ihr Geist so künstlich auszudehnen,

30 Anagram ihres Namens, gemäß der Mode der Zeit. 
Dass eine süsse Klag', ein fast verliebtes Sehnen, Aus todten Sehnen bricht. Gefällt Ihr denn die Eil: So gleicht an Schnelligkeit kein Sturm, kein Stral, kein Pfeil, Kein Blitz, kein Wirbel-Wind den wolgemess'nen Sprüngen, Die Ihrer raschen Hand niemalen missgelingen.

Die Töne Menge bricht, gleichwie ein Storm hervor, Und scheint's, man hör' in Ihr den ganzen Musen-Chor.

Euch, die ihr Sie gehör't, ersuch ich tadelt nicht, Ob hätt' ich Ihren Rum nicht hoch genug getrieben!

Ihr andern, wenn ihr denkt, hier wäre viel erdicht't:

So hör't Sie erst, dann sprecht, ob ich zu viel geschrieben" (Klima 1984, S. 38) (Rathje 1984, S. 112-113)

Brockes' Freundeskreis reagierte auf die Aufforderung, wenn er sich später in der Wohnung der Brockes versammelte, wo Anna Klavier und Laute spielte. Richey, einer der Literaturfreunde, schrieb das folgende Gedicht:

Wir, die wir Sie gehör't, wir tadeln freylich nicht,

Ob hätt'st du Ihren Ruhm, aus Gunst, zu hoch getrieben;

Es hat die Stärke selbst in deinem Lob-Gedicht'

Ein Wunder solcher Kunst noch viel zu schwach beschrieben" (Rathje 1984, S. 113).

Hier ist wieder eine großartige Beschreibung von einem großen Ausübenden, der mit seinem Spiel aufrühren und beruhigen kann und die Zuhörer ganz in seiner Hand hat. Es ist nicht verwunderlich, dass Mattheson von ihrem Spiel begeistert war. Nun müssen wir uns darüber klar sein, dass Brockes ein naher Freund von Mattheson und Telemann war, der Letztere setzte viele Texte von ihm in Musik. Das könnte Matthesons Begeisterung ausgelöst haben, denn er war zur selben Zeit ein Mann der scharfen Zunge und gerader Worte (Farstad 2000, S. 115-120) sowie voller scharfer Kritik, die er an die Lautenisten und die Laute im Barock richtete.

Brockes setzte seine Einfluss als Ratsherr in Hamburg ein, um Georg Philipp Telemann schon 1721 hierherzuholen, und so kann man annehmen, dass es zwischen den Familien von Telemann und Brockes enge Verbindungen gab. Viele Konzerte mit Texten von Brockes und Matthesons Musik wurden in und um Hamburg veranstaltet. Viele davon auch in Brockes Haus (Fry 1980, S. 71 104), welches eine Heimstadt für Musiker war, genau wie dasjenige der Gottscheds in Leipzig. Gewiss hatte Anna Ilsabe beim Arrangieren der Konzerte die Finger mit im Spiel, es gilt als sicher, dass sie selbst dabei Soli oder den Generalbass auf der Laute spielte (siehe das Gedicht von Brockes). Mattheson hatte im übrigen 
eine großen Respekt vor Weiss als Lautenist, aber hielt nicht viel von den anderen Lautenisten seiner Zeit. Eine Ausnahme war wohl Ilsabe, die offenbar doch von Zeit zu Zeit öffentlich auftrat.

In der Zwischenzeit lebte Anna Ilsabe im Alltag das anonyme Leben einer braven Hausfrau und Mutter und hielt sich gerne im "Niedrigen” auf, eine Haltung, die von ihrem Glauben und der Gottesfurcht geprägt war.

\section{Stuttgart}

Maria Dorothea (St. Pierre) Spurnÿ (Spourny) (Tschechien, ca. 1708/1722 bis 1758, Leiden) war mit Franz Spurny verheiratet, der als Hornist in Stuttgart von 1721 bis 1736 angestellt war. Erwähnt wird Maria Dorothea von 1733/34 als Lautenistin. Nach einem kurzen Aufenthalt in Polen waren beide wieder in Stuttgart angestellt und lebten dort von 1744 bis 1755. In dieser Zeit war Maria Dorothea als Lautenvirtuosin angestellt. Joachim Domning vermutet, dass Frau Spurny die Lautenlehrerin von Louise Friederike (ab 1733 Herzogin von Mecklenburg) gewesen ist (Domning 2000, S. 48). Louise Friederike zog 1746 nach Mecklenburg und war dort bis 1756 Herzogin. Ein Lautenbuch trägt ihren Namen «Pieces choisies pour le Lut / Pour / son Altesse serenissime Madame la Princesse Luise de Wurttemberg». Über ihr Lautenspiel ließ sich allerdings in den Archiven Mecklenburgs nichts in Erfahrung bringen, doch muss sie wohl die Musikaliensammlung ihres Vater, des Erbprinzen Friedrich von Württemberg, weiter auch mit Lautenmusik, u. a. von Daube, bereichert haben. ${ }^{31}$

\section{Bayreuth}

Friederike Sophie Wilhelmine von Bayreuth (1709 -1758).

Musik war die größte Passion von Kronprinz Friedrich von Preußen, eine Passion, die er mit seiner Schwester Wilhelmine teilte, wie man es in vielen Briefen, die sie sich in der Zeit zwischen 1728 und 1758 schrieben, weiß (Volz 1924/26). Die Reise nach Dresden im Jahre 1728 war ein Wendepunkt für den zukünftigen König in Berlin (Fürstenau 1971, II, S. 164). Es war ein gewaltiger und fantastischer Eindruck, als Friedrich nach Dresden kam und die besten Aufführenden Europas dieser Zeit hörte und sah. Zwei dieser Musiker waren der Flötist Johann Joachim Quanz und der Lautenist Silvius Leopold Weiss. Als König August zusammen mit Konzertmeister Pisendel, Flötist Buffardin, Johann Joachim Quanz und Silvius Leopold Weiss 1728 Berlin besuchte, blieben Weiss und Quanz für drei Monate dort zum Spielen und Unterrichten. 1731 heiratete Wilhelmine den Thronfolger Friedrich, Sohn des Markgrafen Georg Friedrich Karl von Bayreuth.

1732 zog Wilhelmine nach Bayreuth und setzte dort ihr Lautenspiel fort. Das bekräftigt ein Brief an ihren Bruder vom 17. Oktober 1733:

31 Louise von Würtemberg heiratete 1746 nach Mecklenburg und wurde dort Herzogin. 
"Ich habe wieder Bekanntschaft mit Prinz Dickbauch [ihre Laute], dem ergebenen Diener der Princesa [Friedrichs Flöte], gemacht und ihn so gut gezogen, daß ich Großes leisten werde. Ich fange demnächst mit dem Generalbaß an; mein Lehrer ist sehr tüchtig. Ich will ihn nach Dresden schicken, damit er Weiß hört, und wenn du befiehlst, kann er einen Abstecher nach Ruppin machen!“" (Volz 1924/26 S. 164).

"Ich fange wieder an, meine Finger in Übung zu bringen, um der Prinzessin Flöte den Kleidsaum zu küssen. Der Prinz Dickbauch dreht sich um sie, wie die Erde um die Sonne; er stösst so schmachtende Seufzer aus, dass es erbarmenswürdig anzuhören ist" (Volz 1924/26, S. 113).

Die Namen der Instrumente basieren auf einem Scherz von Friedrich und Wilhelmine. Friedrich gab seiner Flöte den Namen Prinzipessa, was bedeuten könnte, dass er keine andere Prinzessin lieben könnte. Wilhelmine hatte die Laute Prinz Dickbauch getauft, und das erklärt, dass die Laute Friedrichs Rivale wird (Volz 1924/26, S. 69).

"Ohne mich rühmen zu wollen, kann ich sehr gut die Laute schlagen und beginne mit dem Generalbass, der mich fast den ganzen Tag beschäftigt" (Volz 1924/26, S. 308).

Es waren viele Musiker am Hofe angestellt, darunter der Lautenist Adam Falckenhagen (1697-1754), Friedrich - Friederike Sophie Wilhelmines Mann und ab 1735 der neue Markgraf von Bayreuth - war Flötist. Gemeinsam mit Wilhelmine brachte er Geld zusammen, um den Hof zu renovieren und so den musikalischen Ansprüchen der Zeit gerecht zu werden. Von 1735 bis zu ihrem Tode 1758 ließ Wilhelmine das Musikleben und das Theater aufblühen. ${ }^{32}$ Auf dem Gebiet der Musik und der Oper arbeitete Wilhelmine mit ihrem Bruder König Friedrich in Berlin zusammen, der ihr neue Musik und gute Musiker schickte. Musik wurde an Geburtstagen und zum Karneval gebraucht sowie bei fürstlichen Besuchen mit Oper und Kammermusik. In einem Brief vom 28. Februar 1736 schrieb Wilhelmine, dass sie jeden Abend Musik hatten (Volz 1964, S. 311) oder sich abends mit Ball oder Musik vergnügten. "Wir leben hier zufrieden wie die

32 Außer Falckenhagen waren diese Lautenisten in Bayreuth: Paul Charles Durant (1712-?); Bernhard Joachim Hagen (1720-1787). Von vielen Bayreuther Komponisten wurden Werke für Laute komponiert oder bearbeitet: (Jacob Friedrich?) Kleinknecht (1722-1794); Johann Pfeiffer (1697-1761); Johann Balthasar Kehl (17251778); Karl Siegmund Freiherr von Seckendorff (1744-1785). 
Könige und haben fast täglich Ball und Musik” (Volz 1964, S. 166).

Wilhelmine wurde früh mit der Laute und dem Lautenspiel bekannt. In Berlin unterwies sie ein Lautenist namens Feldern. Sie empfing eine besondere Inspiration während des erwähnten Besuches von Silvius Leopold Weiss aus Dresden 1728, und in Bayreuth erhielt sie Unterricht von Adam Falckenhagen. Es ist also nicht unwahrscheinlich, dass sie sein Duetto F-Dur zusammenspielten ${ }^{33}$.

\section{Einige unbekanntere Lautenistinen}

Marie Aurora von Königsmarck zu Quedlinburg (1662-1728) wohnte einige Zeit in Schweden (ihre Mutter war die schwedische Baronesse Maria Christina von Wrangel zu Lindeberg). Nach dem Tode ihrer Mutter 1691 wohnte sie in Quedlinburg. Sie sprach mehrere Sprachen, war eine Virtuosin auf der Laute und der Viola da Gamba, komponierte einige Opern und Gesangstücke sowie Kantaten, aber Lautenkompositionen sind nicht von ihr überliefert (Christensen 2007).

\section{Fürstin Kaunitr}

Der Lautenist Antonius Eckstein lebte ungefähr von 1657 bis 1720. Emil Vogl erwähnt die Möglichkeit, dass er der Lautenlehrer von Fürstin Kaunitz gewesen ist. (Vogl 1964, S. 41-45).

\section{Prinzessinnen von Karl IV.}

Wolfgang Adam Anton Hoffer (?-1757 in Mainz) war im September 1740 als Kammerherr und Lautenist am Mainzer Hof angestellt. Hoffer kam aus Wien (Schweickert 1937, S. 28 / Radke 1972, S. 320-321). Ernst Gottlieb Baron traf Hoffer in Berlin. Marpurg schrieb:

Abgesehen von der Bekanntschaft mit dem berühmten Lautenisten [Weiss] war er [Baron] auch mit Herrn von Hofer aus Wien bekannt, der hatte die Prinzessinnen von Karl IV unterrichtet und ging dann in Kurmainzerische Dienste (Marpurg 1754 bind I, S. 546).

\section{Elisabeth Stricker}

Augustin Reinhard Stricker war 1714 Kapellmeister am Hofe in Köthen. Die Kapelle wurde 1754 aufgelöst (Kraft 1980, S. 828). A. R. Strickers Ehefrau Catherine Elisabeth Stricker war Sängerin und später Lautenistin in Köthen. Das Gehalt, das sie empfing, betrug etwa nur die Hälfte des Lohnes, den ihr Mann bekam. Ab dem frühen 17. Jahrhundert war es üblich, für seine Kinder einen Lautenmeister am Hof zu haben. Es ist gut möglich, dass Elisabeth diese Stellung innehatte.

33 Augsburger Staats- und Stadtbibliothek $2^{\circ}$ Tonkunst, Faszikel III 


\section{Christina Agnes Agnera von Anbalt-Köthen}

Johann Michael Sciuro (Ciurus, Ciurius, Sciurus, Scyurus) (Lebensdaten unbekannt) war auch Lautenist in Köthen, und zwar von 1724 bis 1754. Seine insgesamt 200 Bearbeitungen von Chorälen für Laute befinden sich in zwei Manuskripten der Berliner Staatsbibliothek. Heute liegen sie in Krakau: Ms. 40150; weitere Choräle finden sich auch in Ms. 40151 zusammen mit zwei Sonaten von Friedrich Wilhelm Rust (1739-1796), (Farstad 2000, S. 465). Auf der Frontseite von Sciurius' "Canzoni devotti" finden sich die Initialen C. A. A. Pr. D.'A. / 1740. Das sind die Initialen von Christina Anna Agnera, Prinsesse von Anhalt-Köthen. Es scheint, als ob sie später die Förderin von Friedrich Wilhelm Rust gewesen sei, als er Kapellmeister in Anhalt-Dessau war. Christina studierte Cembalo bei J. S. Bach und Laute bei Sciurius. Es kann gut sein, dass Rust seine Sonaten für sie geschrieben hat (Farstad 2000, S. 463-464). ${ }^{34}$

\section{Schluss}

Baron schreibt das galante plaisante Frankreich welches gemeiniglich auf der Saiten zeigen kan, wo es am schönsten aussiehet, macht durch sein freyes und lebhaftes Wesen die Music mehr schertzend und das ich so rede gleichsam negligent und tendelhaft als alle anderen civilisierten Nationen derowegen sie auch mehr Dames als ernsthafte und reele Gemüter afficirret (Baron 1727/1976, S. 148).

Ernsthaftigkeit und echtes Temperament ist hier als Ausdruck des männlichen Charakters gedacht und vermittelt einen Eindruck von der damaligen Einstellung des Weiblichen als folgsam, einfühlsam für Affekte, Passionen und allgemeine Gefühle, zugleich aber auch seicht und oberflächlich. Diese Ausführungen Barons führen zu den Schlussbetrachtungen über die Stellung der Lautenspielerinnen im Deutschland des 18. Jahrhunderts.

Die meisten uns bekannten Lautenistinnen der damaligen Zeit waren, wie zu sehen war, verbunden mit den aristrokratischen Kreisen, denn die meisten Frauen, die eine gute Ausbildung hatten, waren Prinzessinnen oder Ehefrauen von Männern, die mit dem Adel verbunden waren oder sonst zur Oberschicht gehörten. Der Artikel zeigt, dass viele dieser Frauen ausgezeichnete Instrumentalistinnen waren und die Laute auf einem sehr hohen Niveau spielten. Es ist indessen charakteristisch, dass es keine hinterlassenen Kompositionen von diesen Frauen gibt. Nur Ilsabe Brockes und Adelgund Gottsched intabulierten, soweit wir wissen, Musik für Laute, und Letztere regte andere Frauen an, dies auch zu tun. Warum komponierten sie nicht für ihr Instrument, und warum waren sie so wenig sichtbar im öffentlichen Raum, speziell als mitwirkende Lautenistinmen im

$34 \quad$ Notizen zu Elisabeth Stricker und Christina Anna Agnera, Prinzessin von Anhalt-Köthen, schrieb der Lautenforscher Arthur Ness, USA, in einem Brief vom 9. April 2007 nieder. 
Solo oder Zusammenspiel? Weder Gottsched, Ziegler, Lehmann, Wilhelmine von Bayreuth oder Wilhelmina von Lobkowitz waren in der öffentlichen Konzertszene aktiv, selbst wenn man sie aus heutiger Sicht vom Niveau her als professionelle Musikerinnen ansehen könnte. Aber Frauen durften sich eben in der Öffentlichkeit nicht produzieren. Sie führten ihre Kunst mehr im Verborgenen und im privaten Bereich aus oder als täglichen Zeitvertreib (z. B. Wilhelmine von Bayreuth). Wie wir bereits gesehen haben, war das Dasein der Frauen so geregelt, dass es eine Entwicklung oder Fortschritt zur Komponistin nicht gab. Das wird deutlich an Anna Ilsabe Lehmann Brockes' Leben, die von ihrem Mann als die perfekte Hausfrau, die die Kinder aufzieht und das Haus in Stand hält, beschrieben wird. Dasselbe kann man an Luise Adelgunde Gottsched sehen, die ihre eigenen Anlagen als Musikerin und Lautenistin aufgab, um sich in den Dienst für ihren Mann zu stellen. Eine Künstlerin zu sein, war unvereinbar mit den Pflichten einer Hausfrau. So kann man nur feststellen, dass, obwohl es viele Frauen gab, die Laute spielten, nur wenige im Licht der Öffentlichkeit auftraten. Wie wir bereits gesehen haben, war das Frauendasein so geregelt, dass es eine Entwicklung oder Fortschritt zur Komponistin nicht gab. Die wichtigsten Kriterien waren: Sie mussten aus einer Familie mit einer Musiktradition stammen, eine gute Musikausbildung genossen haben, sie waren finanziell unabhängig, hatten keine Mühe mit hausfraulichen Pflichten und gute Kontakte zur Musikszene. Das waren Voraussetzungen, die die meisten Frauen, vor allem aus den niederen Schichten, nicht erfüllten. Für die Laute hatte das allerdings wohl auch positive Folgen, denn weil diese Instrumente erst mit Hilfe der elektronischen Verstärkung in größerem Rahmen eingesetzt werden konnten, blieb der Salon und die „Kammer“ als Refugium lange erhalten und stellte mit dem Übergang zur Gitarrenmusik eine Traditionslinie her, die bis in unsere Zeit reicht. Die „moderne“ Musik ging andere Wege, ohne die Laute. Wenn auch nicht immer greifbar, so riss das Interesse an dem angerissenen Saitenton seit der Erfindung des mit einer Sehne gespannten Bogens in der Steinzeit nie ab. Es mag manchem Lautenliebhaber heute davor grauen, mit der „Starkstromgitarrre“ auf eine Ebene gestellt zu werden. Der Geschichte ist das egal. Und wenn Frauen heute auf der Bühne die Saiten zum Schwingen bringen, dann haben Frauen wie Adelgunde Gottsched und ihre Schwestern den Weg dazu bereitet.

\section{Per Kjetil Farstad \\ University of Agder}

\section{Literatur:}

Baron, Ernst Gottlieb 1976: Study of the Lute, Instrumenta Antiqua 1976, English-ûbersetzung von Douglas Alton Smith von Historisch-Theoretisch und Practische Untersuchung des Instruments der Lauten, Nürnberg, 1727.

Buelow , George J. 2007: 'Johann Christoph Gottsched” Grove Music 
Online ed. L. Macy http://www.grovemusic.com, Zugang 21 Mars 2007.

Crawford, Tim 1986, 'Lord Danby's Lute Book, A New Source of Händel's Hamburg Music”, in Göttinger Händel-beiträge 2, S. 19-50.

Christensen, Martin K. I. 2007 "Worldwide Guide to Women in leadership", http:/ / www.guide2womenleaders.com, Zugang 25. März 2007.

Det Danske Sprog- og Litteraturselskab, 2005-7, Ordbog over det Danske Sprog - dansk i perioden 1700-1950 http://ordnet.dk/ods/opslag?id=435275, Zugang 25. mars 2007.

Domning, Joachim 2000: "Zur Faksimile-Ausgabe des Rostock Lautentabulaturen-Faszikels Mus. Saec. XVIII 65.6 a-z”, in: Die Laute nr. III, S. 75-93.

Dreyfus, Laurence 1987: Bach's Continuo Group, Cambridge.

Erondelle, Pierre 1989: The French Garden: for English Ladyses and Gentlewomen to walk in, or a Sommer Dayes Labour, London, 1601, Austern, 1989. Sitert und Tick, Judith und Ericson, Margaret in: II. Western classical traditions in Europe and the USA http://www.grovemusic.com, Zugang 25. mars 2007.

Farstad, Per Kjetil 2005: "Johann Sebastian Bach and the lute", Festschrift für Olle Edström, Skrifter fra institutionen för musikvetenskap, Göteborgs universitet nr. 80, S. 14-30.

Farstad, Per Kjetil 1998: "Ornaments in 18th Century German Lute Music", Die Laute nr. II, 1998, S. 85-115.

Farstad, Per Kjetil 2000: German Galant Lute Music in the 18th Century, Dissertation Nr. 58, Göteborg Universitet.

Farstad, Per Kjetil 2001: "Some new Biographical Information on Bernhad Joachim Hagen" in: Journal of The Lute Society of England, nr. 40.

Farstad, Per Kjetil 2002: "The Life and Works of Ernst Gottlieb Baron (1696-1760)", in: Journal of The Lute Society of America, nr. XXX 1997, trykt i 2002, S. 42-82.

Forkel, Johann Nikolaus 1802: Über Johann Sebastian Bachs Leben, Kunst und Kunstwerke, Leipzig.

Flotzinger, Rudolf 1986: "Rochus Berhandtsky und Wolff Jacob Lauffensteiner, zum leben und Schaffen zweier Lautenisten in kur-bayerischen Diensten”, Studien für Musikwissenschaft (StMw), XXVII).

Fry, Harold P. 1980: "Barthold Heinrich Brockes und die Musik, von Barthold Heinrich Brockes (1680-1747). Dichter und Ratsherr in Hamburg, Neue Forschungen zu Persönlichkeit und Wirkung", herausgegeben von Hans-Dieter Loose, Hamburg, S. 71-104.

Fürstenau, Moritz 1971: Zur Geschichte der Musike und des Theaters am Hofe zu Dresden, Vol. I und II, Reprint der Ausgabe von 1861-1862 Georg Olms, New York. 
Gottsched, Luise Adelgunde Victoria von 1763: Der Frau Luise Adelgunde Victoria Gottschedinn, geb. Kulmus, samtliche Kleinere Gedichte, Johann Christoph Gottsched ed., Leipzig: Breitkopf.

Grove Music Online ed. L. Macy, http://www.grovemusic.com, Zugang 21. Mars 2007.

Hensel, J. D. 1787: System der weiblichen Erziehung, bes. f. den mittleren u. höheren Stand, Halle.

Klima, Josef 1984, MariaEnzersdorf bei Wien, "Das Jahrhundert der Barocklaute". Marginalen zur Geschichte der Laute, Wiener Lautenarchiv Sonderband S. 36-38.

Kosack, Hans-Peter 1935: Geschichte der Laute und Lautenmusik in Preussen, Kassel

Koskoff, Ellen 2007: "Women in music, 『III: World music, 3. Music in the court and harem", http://www.grovemusic.com, Zugang 3. april 2007.

Kraft, G.: "Cöthen," The New Grove 1980 (Ausgabe 1995) Dictionary of Music and Musicians, ed. Stanley Sadie, London.

Lappenberg, Johann Martin 1847, Selbstbiographie des Senators Barthold Heinrich Brockes, Band 2.

Lauson, Johann Friedrich 1753: Erstes versuch in Gedichten, Königsberg.

Mace, Thomas 1676: Musick's Monument, London.

Marpurg, Friedrich Wilhelm 1754: Historish-kritische Beyträge zur Aufnabme der Musike im 5. Band, Berlin: G. A. Lange, 1754, 1762, und 1778.

Meyer, Christian 1997: Sources Manuscrites en Tablature Luth et Theorbe (c. 1500c.1800), Vol III/1, Ôsterreich (A), Baden-Baden \& Bouxwiller, Édtions Valentin Koerner, 1997.

Mattheson, Johann 1713: Das Neu-Eröffnete Orchestre, Hamburg.

Neemann, Hans 1939: "Die Lautenistenfamilie Weiss", Archiv für Musikforschung, Leipzig.

Nettl, Paul 1922-1923: "Zur Geschichte des Konzertwesens in Prag”, ZfM, Vol.V.

Radke, Hans 1972: "Kompositionen des Lautenisten Wolffgang Adam Anton Hoffer", Mitteilungen der Arbeitsgemeinschaft für mittelrheinische Musikgeschichte, Mainz.

Rathje, Jürgen 1984: "Zur hamburgischen Gelehrtenrepublik im Zeitalter Matthesons”. Artikkel i New Mattheson Studies, herausgegeben von George J. Buelow und Hans Joachim Marx, Cambridge.

Reilly, Edward R., Smith, Douglas Alton, Crawford, Tim 2007-2011: "Sylvius Leopold Weiss", Oxford Music Online, zugang 21. April 2010, http://www. oxfordmusiconline.com/subscriber/article/grove/music/30065pg2\#S30065.2.

Rieger, Eva 1988: Frau, Musik und Männerherrschaft. Zum Ausschluss der Frau aus der deutschen Musikpädagogik, Musikwissenschaft und Musikausübung, 2. Auflage, Kassel. 
Lautenistinnen in Deutschland im 18. Jahrhundert

Rifkin, Joshua und Küster, Konrad 2007: "Christiane Mariane von Ziegler" Grove Music Online ed. L. Macy, http://www.grovemusic.com, (Zugang 21. Mars 2007).

Sadie, Stanley ed. 1980: The New Grove (Auflage 1995) Dictionary of Music and Musicians, London.

Sander, Ina 1966: "Johann Pfeiffer, Leben und Werk des letzten Kapellmeisters am Markgraflichen Hof zu Bayreuth”. Archiv für Geschichte von Oberfranken, bind. 46, Bayreuth, S. 129-181.

Schering, Arnold 1941. Der Musikgeschichte Leiprigs, Band III, 1725-1800, Leipzig

Schletterer, H.M. 1864: Joh. Friedrich Reichardt, Sein Leben und seine musikalische Thätigkeit, Leipzig, S. 77.

Schleuning, Peter 1984: Das 18. Jahrbundert: Der Bürger erhebt sich, Hamburg.

Schoenfeld, Hermann 1908: "Women of the Teutonic Nations Philadelphia", Rittenhouse Press, S. 278-282.

Schusky, Renate, 1997, aus dem Vorlesemanuskript „Kräht ja doch kein Hahn danach ...?" Weibliche Komponistinendes 18. und 19. Jahrhundert, von Dr. Renate Schusky, 10. März 1997, FernUniversität Gesamthochschule Hagen, Zugang 21. mars 2007. https://www.fernunihagen.de/GLEICHSTELLUNG/pdfDateien/ Schusky.pdf

Schweickert, Karl 1937: Beiträge zur Geschichte der Stadt Mainz, Band 11, "Die Musikpflege am Hofe der Kurfürsten von Mainz im 17. und 18. Jahrhundert”, Mainz.

Smith, D. A. 1973, "Baron and Weiss contra Mattheson: In defense of the lute", Artikkel in JLSA, nr. VI, S. 48-62.

Smith, Douglas Alton 1977: The Late Sonatas of Silvius Leopold Weiss, Dissertation, Stanford University.

Thomsen-Fürst, Rüdiger 2002: "Zur Biographie der Juliana Margaretha und zu einem unbekannten Zweig der Lautenistfamilie Weiss", in Die Laute (Jahrbuch der Deutschen Lautengesellschaft) Nr. IV, Frankfurt a. M., S. 41-47.

Tick, Judith og Ericson, Margaret 2007: II. "Western classical traditions in Europe and the USA". http://www.grovemusic.com, Zugang 25. mars 2007.

Vogl, Emil 1964: "Aureus Dix and Antoni Eckstein, zwei Prager Lautenisten." Die Musikforschung, Band. XVII/I, S. 41-45.

Vogl, Emil 1972: "Eine Kirchenmusik mit Lautebegleitung in Böhmen." Hudební véda IX/1, S. 42-48.

Volkmann, Hans 1906/1907: "Sylvius Leopold Weiss / Der Letzte grosse Lautenist", Die Musik 6, s. 274-289.

Volz, Gustav Berthold 1924/26; Friedrich der Grosse und Wilhelmine von Bayreuth, Band I: Jugendbriefe, 1728-1740; Band II: Briefe der Königzeit, 1740-1758, Berlin and Lepzig, 1924 und 1926.

Walther, Johann G. 1732, Musikalisches Lexicon, Leipzig.

Aasen, Henriette Sinding, 2007: "Kvinner ikke med da rettsstaten ble til", Interview von 2003 in http://kilden.forskningsradet.no, Zugang 4. april 2007. 